\title{
Bisphosphonates in the treatment of Charcot neuroarthropathy: a double-blind randomised controlled trial
}

\author{
E. B. Jude ${ }^{1}$, P. L. Selby ${ }^{1}$, J. Burgess ${ }^{1}$, P. Lilleystone ${ }^{2}$, E. B. Mawer ${ }^{1}$, S. R.Page ${ }^{2}$, M. Donohoe ${ }^{3}$, A. V.M. Foster ${ }^{4}$, \\ M.E.Edmonds ${ }^{4}$, A.J.M.Boulton ${ }^{1}$ \\ ${ }^{1}$ Department of Medicine and Diabetic Foot Clinic, Manchester Royal Infirmary, Oxford Road, Manchester, UK \\ ${ }^{2}$ Queens Medical Centre, Nottingham, UK \\ ${ }^{3}$ Royal Devon and Exeter Hospital, Exeter, UK \\ ${ }^{4}$ King's Hospital, London, UK
}

\section{Abstract}

Aims/hypothesis. The management of charcot neuroarthropathy, a severe disabling condition in diabetic patients with peripheral neuropathy, is currently inadequate with no specific pharmacological treatment available. We undertook a double-blind randomised controlled trial to study the effect of pamidronate, a bisphosphonate, in the management of acute diabetic Charcot neuroarthropathy.

Methods. Altogether 39 diabetic patients with active Charcot neuroarthropathy from four centres in England were randomised in a double-blind placebo-controlled trial. Patients received a single infusion of $90 \mathrm{mg}$ of pamidronate or placebo (saline). Foot temperatures, symptoms and markers of bone turnover (bone specific alkaline phosphatase and deoxypyridinoline crosslinks) were measured over the 12 months, in 10 visits. All patients also had standard treatment of the Charcot foot.

Results. Mean age of the study group (59\% Type II (non-insulin-dependent) diabetes mellitus) was $56.3 \pm 10.2$ years. The mean temperature difference between active and control groups was $3.6 \pm 1.7^{\circ} \mathrm{C}$ and $3.3 \pm 1.4^{\circ} \mathrm{C}$, respectively. There was a fall in tem- perature of the affected foot in both groups after 2 weeks with a further reduction in temperature in the active group at 4 weeks (active and placebo vs baseline; $p=0.001 ; p=0.01$, respectively), but no difference was seen between groups. An improvement in symptoms was seen in the active group compared with the placebo group $(p<0.001)$. Reduction in bone turnover (means \pm SEM) was greater in the active than in the control group. Urinary deoxypyridinoline in the pamidronate treated group fell to $4.4 \pm 0.4 \mathrm{nmol} / \mathrm{mmol}$ creatinine at 4 weeks compared with $7.1 \pm 1.0$ in the placebo group $(p=0.01)$ and bone-specific alkaline phosphatase fell to $14.1 \pm 1.2$ $\mathrm{u} / \mathrm{l}$ compared with $18.6 \pm 1.6 \mathrm{u} / \mathrm{l}$ after 4 weeks, respectively $(p=0.03)$.

Conclusion/interpretation. The bisphosphonate, pamidronate, given as a single dose leads to a reduction in bone turnover, symptoms and disease activity in diabetic patients with active Charcot neuroarthropathy. [Diabetologia (2001) 44: 2032-2037]

Keywords Charcot neuroarthopathy, diabetes mellitus, bisphosphonates, foot temperature, bone turnover markers.
Charcot neuroarthropathy (CNA) is a disabling and devastating condition affecting patients with severe

Received: 20 April 2001 and in revised form: 26 June 2001

Corresponding author: Dr. E. B.Jude, Department of Medicine, Manchester Royal Infirmary, Oxford Road, Manchester M13 9WL, UK, email: ejude@dc.cmht.nwest.nhs.uk Abbreviations: CNA, Charcot neuroarthropathy; DPD, deoxypyridinoline; BSAP, bone-specific alkaline phosphatase; VPT, vibration perception threshold diabetic neuropathy. It can lead to foot deformity, recurrent foot ulceration and ultimately to amputation. The incidence is about $0.1-5 \%$ in diabetic patients with peripheral neuropathy [1].

The pathogenesis of CNA is not clear, one possible predisposing factor is reduced bone density [2,3], probably as a result of increases in bone turnover. Investigators measured carboxy-terminal telopeptide domain of type 1 collagen (1TCP) and carboxy-terminal propeptide of type 1 collagen $(\mathrm{P} 1 \mathrm{CP})$ as possible 
markers of bone resorption and bone formation, respectively, in diabetic patients with acute and chronic Charcot arthropathy, diabetic patients without bony involvement and control subjects [4]. They found an increase in 1CTP (but not P1CP) in patients with acute Charcot. Similarly, we showed an increase in bone specific alkaline phosphatase (BSAP) and dehydroxypyridinoline (DPD) in patients with acute Charcot arthropathy [5].

No proven treatment of this condition is available. Current management is immobilization and off-loading in a total contact, air cast or scotch cast boot $[6,7]$. Immobilization for a prolonged period - at times up to a year - can be required [1].

Presently no pharmacological treatment is available for the treatment of CNA. A single centre, open-labelled study suggested the potential benefit of pamidronate, a bisphosphonate, in reducing disease activity and bone turnover markers [8]. In that study a beneficial effect of pamidronate on foot temperature and alkaline phosphatase was observed. In the present study we investigated the efficacy of pamidronate in patients with acute CNA in a double-blind randomised controlled trial.

\section{Subjects and methods}

Study design. The study was carried out in four centres in the United Kingdom and all investigators were trained by the coordinating centre (Manchester). The protocol was approved by the ethics committee in each centre, and patients gave their written, informed consent. Patients were assessed at baseline, two-weekly intervals for the first 3 months and at 6,9 and 12 months thereafter.

Selection of patients. Patients were recruited from four diabetes centres in the United Kingdom (Manchester, London, Nottingham and Exeter). Patients included in the study had Type I (insulin-dependent) or Type II (non-insulin-dependent) diabetes mellitus and were between 20 and 80 years of age. All patients had a definite diagnosis of active Charcot neuroarthropathy (see below) and peripheral neuropathy according to the criteria used.

Assessment of neuropathy. Patients underwent a detailed clinical history and examination, and Neuropathy Disability Score (NDS) were measured [9]. All patients had an NDS score of more than 6. Vibration preception threshold was expressed as a mean of three readings on a biothesiometer (Biomedical Instrument, Newbury, Oh, USA) on the hallux and medial malleoli of both lower limbs, and neuropathy was defined as a VPT of greater than two standard deviations above the age-related mean [10].

Criteria for diagnosis of Charcot neuroarthropathy. The diagnosis of Charcot neuroarthropathy was made on the basis of clinical and radiological examination. Clinical criteria included the presence of a hot, swollen foot with or without redness of the overlying skin. Temperature was measured using an infrared thermometer (Mikron, NJ, USA), at the site of maximum deformity and temperature on the affected foot and a similar site on the contralateral foot. An increase of $2^{\circ} \mathrm{C}$ or more com- pared to the similar site on the contralateral foot was taken to indicate active disease [11]. Foot temperatures were measured at 30, 60 and 90 min after the patients' shoes and socks were removed and an average of three readings was taken (results are presented as the difference between the active and contralateral foot). Patients were asked to rest supine for the duration of the measurements. Patients with a clinical diagnosis of Charcot neuroarthropathy had plain radiographs of the feet and a three-phase technetium-99m-labelled bisphosphonate bone scan.

All patients had standard foot care using either scotch cast boot, pneumatic walker (Aircast, London, UK) or total contact cast to off load the affected foot. None of the patients had peripheral vascular disease (defined as the absence of two or more foot pulses and an ankle brachial index of less than 0.9 ) or renal failure (serum creatinine $>140 \mu \mathrm{mol} / \mathrm{l}$ ).

Pamidronate/placebo infusion. Patients were randomly assigned treatment with Pamidronate ( $90 \mathrm{mg}$ in normal saline) or placebo (matching normal saline) to run over $4 \mathrm{~h}$. The Pamidronate infusion was made up at the pharmacy of each participating centre and the investigators were blinded to the randomisation. A site-specific, computer-generated randomisation list was provided in advance to the study pharmacist at each site, who prepared the medication on the morning of the infusion. Other study personnel, including the patients and investigators, were not aware of the treatment assignments.

Clinical assessment. All patients were followed up at two week intervals for the first three months and then at 6,9 and 12 months. At each visit foot temperatures were measured as at baseline and at the same site. Symptoms related to the Charcot foot were assessed at baseline and patients were asked to score them on a scale of 1 to 10 . Each patient was asked to quantify pain, discomfort and swelling in the foot. These scores were added to give an overall symptom score for that time point. This was then repeated at each 3 monthly visits $(3,6,9$ and 12 months). None of the patients had foot ulcers at entry in to the study.

Bone-turnover markers and biochemical analysis. Blood from an antecubital vein was collected after an overnight fast and after resting for $15 \mathrm{~min}$ in the supine position. At each visit patients had fasting glucose (Glucose oxidase, Boehringer Mannheim, Mannheim, Germany), glycated haemoglobin (HPLC, Diamat, BioRad, Calif., USA), serum creatinine, calcium and alkaline phosphatase (Hitachi 747, Hitachi, Japan) concentrations estimated.

Bone turnover markers, DPD crosslinks and bone specific alkaline phosphatase (BSAP), were measured in all patients. Fasting blood (for BSAP) and fasting, second-voided urine (for DPD crosslinks) were collected at baseline and each follow-up visit. All samples were collected between 9:00 and 10:00 $\mathrm{h}$ to minimise interference with circadian changes. Serum was separated within $1 \mathrm{~h}$ from collection and kept frozen at $-80^{\circ} \mathrm{C}$ until analysed. Urine volume was measured and then urine samples aliquoted and stored without preservative at $-20^{\circ} \mathrm{C}$. DPD crosslinks and BSAP were estimated using kits from Metra Biosystem (Mountain View, Calif., USA). The intra- and interassay coefficient of variation for BSAP was $5.0 \%$ and $6.2 \%$, respectively; and for DPD crosslinks was $6.1 \%$ and $6.8 \%$, respectively.

Statistical analysis. Results are shown as means \pm SEM. Temperatures are shown as the difference between the Charcot and the contralateral foot. The changes in temperature, symp- 


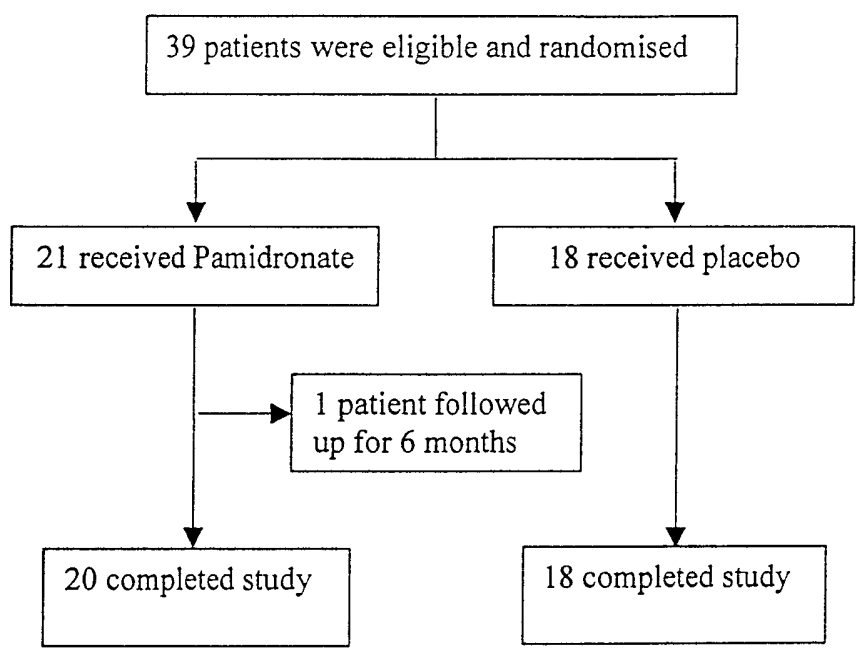

Fig. 1. Flow chart of patients in the study

toms and biochemical markers of bone turnover were compared using a repeated measures general linear model with $t$ tests to determine post hoc differences. For temperature and symptom score the area under the curve were plotted against time and calculated using the trapezium rule. This gives an overall measure of the effect of therapy across the treatment period. Power calculations suggested that 38 patients would be necessary to detect a reduction in temperature differential of less than $2{ }^{\circ} \mathrm{C}$ in the active group with $80 \%$ power and $5 \%$ significance assuming no change in the control group. All analyses were done using the Statistical Package for Social Sciences (SPSS, Chicago, Ill., USA). A $p$ value of less than 0.05 was considered to be statistically significant.

\section{Results}

Altogether 39 patients (26 males, 13 with Type I diabetes, mean \pm SD, age $56.3 \pm 10.2$ years) with a diagnosis of active Charcot arthropathy were included in the study: 16 from Manchester, 12 from Nottingham, 8 from London and 3 from Exeter). Of those, 21 patients received Pamidronate and 18 patients, placebo (Fig.1). Of the 39 patients, only one patient (in the active group) did not continue the study beyond 6 months, his data being included in the analyses until his last visit. There was no difference in age, duration of diabetes, renal function or glycaemic control between the active and placebo groups. There was no difference in VPT or NDS or presence or absence of other complications between groups (Table 1). Treatment was tolerated well and only one patient suffered transient myalgia soon after the infusion of pamidronate.

Disease activity and symptom score. The right foot was affected in 22 patients with the midfoot joints most frequently involved. The mean baseline temperature difference in the active and placebo groups was $3.6 \pm 1.7$ and $3.3 \pm 1.4^{\circ} \mathrm{C}$, respectively $(p=0.5)$.

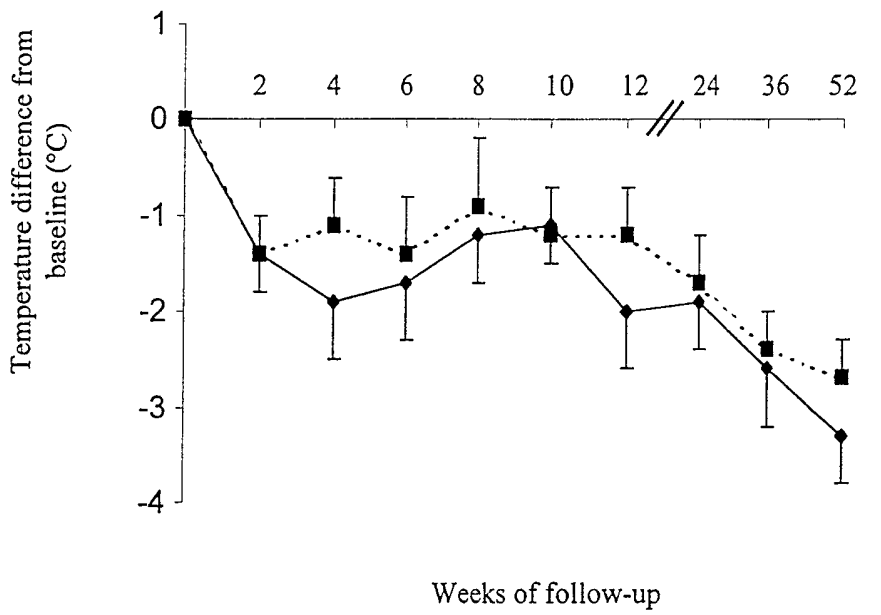

Fig. 2. Effect of Pamidronate on disease activity in patients with Charcot arthropathy. Active group, $\rightarrow$; Placebo group, $\cdots \square \cdots$

Table 1. Baseline clinical and biochemical characteristics of the study subjects

\begin{tabular}{lll}
\hline & Active group & Placebo group \\
\hline$n$ & 21 & 18 \\
Age (years) & $55.9 \pm 10.1$ & $56.5 \pm 8.6$ \\
Duration of diabetes & $17.0 \pm 9.7$ & $19.3 \pm 12.5$ \\
Type II diabetes & 15 & 10 \\
Sex (M/F) & $15 / 6$ & $11 / 7$ \\
NDS & $8.9 \pm 1.2$ & $9.4 \pm 1.1$ \\
VPT & $42.5 \pm 8.9$ & $38.4 \pm 9.1$ \\
Temperature difference & $3.6 \pm 1.7^{\circ} \mathrm{C}$ & $3.3 \pm 1.4^{\circ} \mathrm{C}$ \\
Retinopathy & 15 & 16 \\
Scotch cast boot & 13 & 12 \\
Aircast & 4 & 3 \\
TCC & 3 & 2 \\
Special shoes & 1 & 1
\end{tabular}

$N D S$ neuropathy disability score, $V P T$ vibration perception threshold, TCC total contact cast

There was a significant reduction in temperature in both groups (Fig. 2), greater in the active group compared with the placebo group $[-1.9 \pm 0.8,(p<0.001)$ and $-1.4 \pm 0.7, \quad(p<0.01]$; respectively $)$ at four weeks. There was further temperature reduction throughout the study but no differences were seen between the active and placebo groups at all time points.

The most common symptom was pain followed by discomfort in the foot (Fig. 3). Both groups showed a reduction in symptom score at 3 months. The symptoms in the control group remained constant over the next 12 months, but in the active group a further reduction in symptoms score was seen $(p<0.01)$. Using the area under the symptom score curve as an integrated measure of symptomatology over the whole 12 months of treatment, there was a significant benefit observed with active treatment $(14.3 \pm 8.7$ and $23.8 \pm 8.4$; active vs placebo, $p=0.01$ ). 


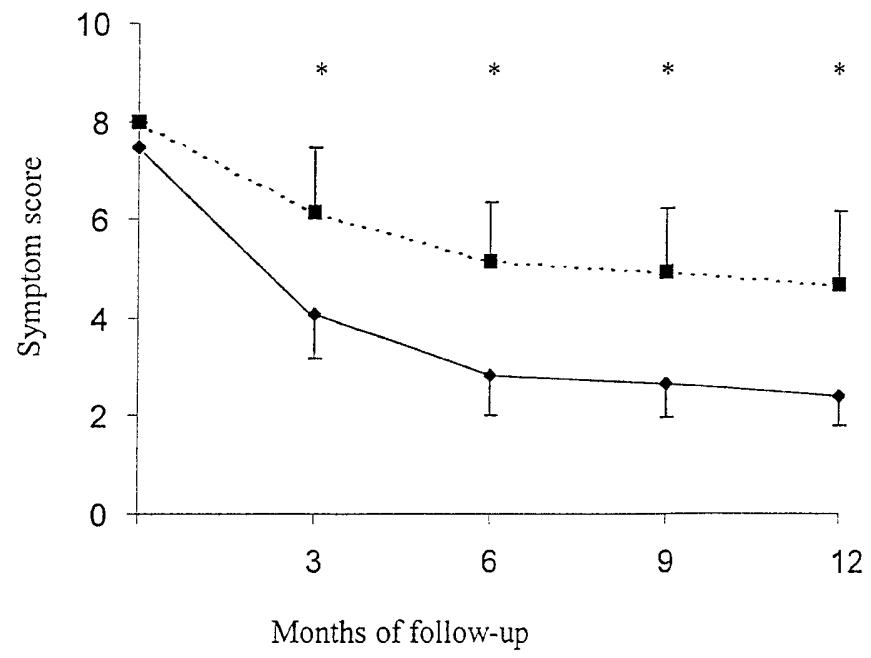

Fig. 3. Effect of Pamidronate on symptoms in patients with Charcot arthropathy. ${ }^{*} p<0.001$. Active group, $\rightarrow-$; Placebo group, $\cdots \square \cdots$

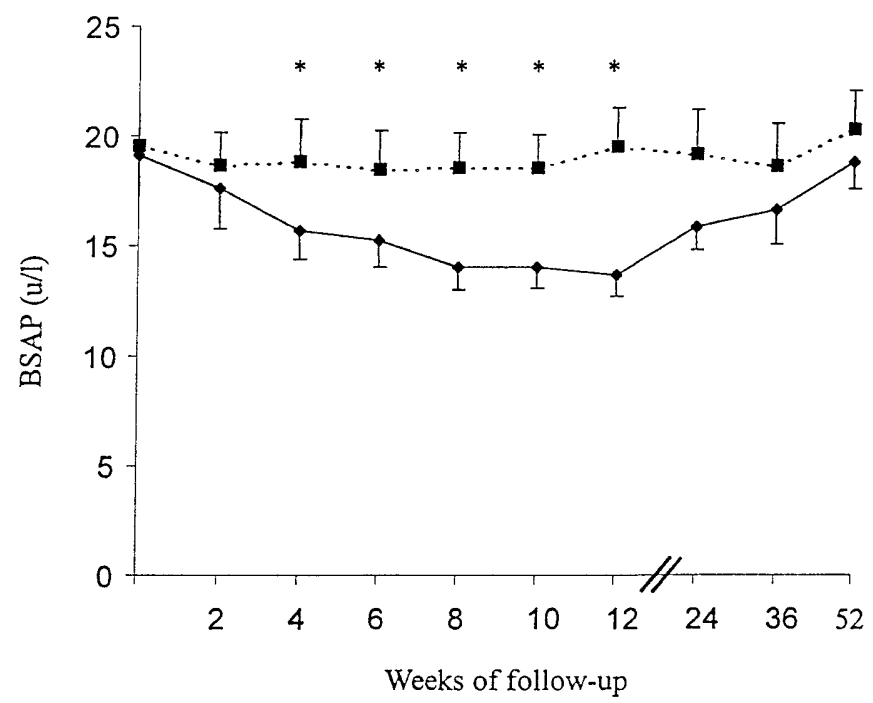

Fig. 4. Effect of Pamidronate on bone specific alkaline phosphatase (BSAP) in patients with Charcot arthropathy. ${ }^{*} p<0.03$. Active group, $\rightarrow-$; Placebo group, $\cdots \square \ldots$

Bone turnover markers. The bone turnover markers measured were bone specific alkaline phosphatase (an indicator of bone formation) and DPD crosslinks (an indicator of bone resorption). No differences were seen at baseline in both markers of bone turnover. The BSAP was significantly reduced in the active group when compared to the placebo group $(p<0.03$, Fig. 4$)$. The difference was observed at 4 weeks and remained so until 24 weeks. Similarly, DPD crosslinks were reduced in the active group at 4 weeks $(p<0.01$ vs placebo, Fig. 5). However, both markers gradually rose towards baseline at the end of the study. Therefore there was a maximum reduction of $32 \%$ and $30 \%$ in DPD and BSAP after Pamidronate infusion.

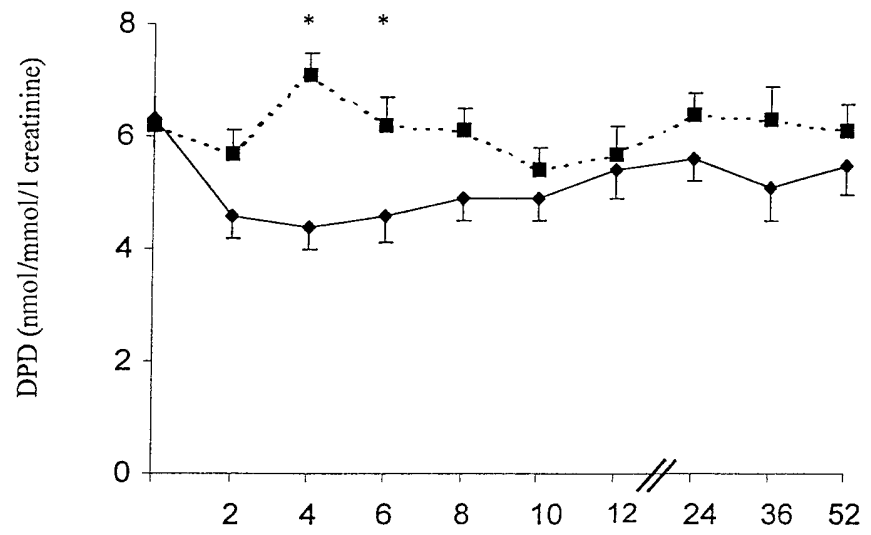

Weeks of follow-up

Fig. 5. Effect of Pamidronate on deoxypyridinoline (DPD) crosslinks in patients with Charcot arthropathy. $* p<0.01$. Active group, $\rightarrow-$; Placebo group, $\cdots \square \cdots$

Inflammatory markers. Erythrocyte sedimentation rate (ESR) and C-reactive protein (CRP) were measured at baseline and at each subsequent visit. There was no difference in ESR and CRP at baseline. No change was seen in ESR or CRP between groups during follow-up.

\section{Discussion}

This is the first study of a definitive treatment for acute CNA as there has previously been no effective treatment to prevent development or progression of this condition. The current treatment recommended for CNA includes bed rest and off-loading of the affected foot using either total contact casting or scotch cast boot $[7,12]$.

There are some similarities in the underlying pathogenesis of CNA and reflex sympathetic dystrophy (RSD). In the latter condition the pathogenesis involves loss of bone density with pain as a predominant symptom, and as Pamidronate was shown to be beneficial [13-15], we did a pilot study of this therapy in CNA with encouraging results [8]. Pamidronate has been shown to be effective in other bone disorders and treatment has shown reduction in bone turnover markers in Paget's disease, and in bone malignancy. It also reduces the development of skeletal fractures in patients with multiple myeloma [16]. In this study of acute CNA, Pamidronate reduced disease activity as measured by skin temperatures over the affected joints, as well as bone destruction, measured indirectly by estimating plasma and urinary bone turnover markers. Measuring foot temperatures has been shown to be an effective means of assessing activity of the Charcot foot [6]. In a recent study of acute CNA, skin temperature was shown to correlate 
with bone uptake of radio-isotope and disease activity [17]. In the present study we found that foot temperatures dropped significantly in the active and placebo groups as early as two weeks after entering into the study. The reduction in temperature in the placebo group is most likely due to the fact that all patients received off-loading measures in agreement with the observations of investigators that following total contact casting significant reduction in skin temperature was observed as well as reduction in bone isotope uptake [17]. In addition to the initial fall in temperature, we also found a further decrease in foot temperatures in the active group. In the placebo group, the temperature remained constant after the initial fall and then decreased over the last 6 months. This is in agreement with the natural history of CNA [6], and is probably due to an improvement in the CNA from the offloading, which was not taken into account in the power calculation. It is possible that if total contact casting had been used in all patients a further decrease and more rapid fall in foot temperatures might have been seen.

The most common symptom of CNA is pain despite the fact that these patients have severe neuropathy $[6,18]$. In our study, pain and discomfort were the predominant symptoms, and both groups showed a dramatic reduction in symptoms two weeks after entering into the study supporting immobilisation as an effective treatment of CNA. However, we did find that patients who received the active treatment showed a further significant reduction in symptoms over time, a reduction which was maintained till the end of the study. This could be as a result of reduction in inflammation with the immobilization, as well as a decrease in cytokine production within the bones of the foot in patients who received the active treatment [19].

One factor in the pathogenesis of CNA is a reduction in bone density associated with an increase in bone turnover markers [4,5]. In this study, we measured DPD and BSAP at each visit during followup. Patients who were treated with Pamidronate had a lower amount of bone turnover markers than the placebo group. This was seen as early as 2 weeks after the Pamidronate infusion, and a significant reduction was seen at 4 weeks. The reduction in DPD lasted for 4 weeks but the decrease in BSAP persisted for up to three months. Previous studies in other bone diseases have also reported similar effects after treatment with bisphosphonates, especially in Paget's disease, where bone turnover markers remain suppressed for up to a median of 36 months [20]. A study done in normal healthy subjects demonstrated a reduction in urinary hydroxyproline but an increase in alkaline phosphatase [21]. We showed a similar reduction in urinary DPD crosslinks but also a simultaneous decrease in bone specific alkaline phosphatase.
This study has thus shown a reduction in bone turnover markers, disease activity and an improvement in symptom scores in the Pamidronate-treated group. The overall reduction in temperature difference was not significant between the active and placebo groups, despite the fact that the actively-treated group showed a further reduction in temperature over time.

In conclusion, Pamidronate has a substantial effect on symptoms in acute CNA, over and above that seen as a result of off-loading. There was also an effect on bone turnover markers. However, the reduction in bone markers was for a limited period only and repeated doses of Pamidronate could be more effective. Further larger studies are needed to investigate the optimal means of using bisphosphonates to treat acute Charcot neuroarthropathy especially the longterm problems of ulceration and deformity.

Acknowledgements. This study was funded by the British Diabetic Association and National Health Service Research and Development Levy.

\section{References}

1. Sanders LJ, Frykberg RG (1991) Diabetic neuropathic osteoarthropathy: The Charcot foot. In: Frykberg RG (ed) The high risk foot in diabetes mellitus. Churchill Livingstone, New York, pp 297-338

2. Young MJ, Marshall A, Adams JE, Selby PL, Boulton AJM (1995) Osteopenia, neurological dysfunction, and the development of Charcot neuroarthropathy. Diabetes Care 18: 34-38

3. Childs M, Armstrong DG, Edelson GW (1998) Is Charcot arthropathy a late sequela of osteoporosis in patients with diabetes mellitus? J Foot Ankle Surg 37: 437-439

4. Gough A, Abraha H, Purewal TS et al. (1997) Measurement of markers of osteoclast and osteoblastic activity in patients with acute and chronic diabetic Charcot neuroarthropathy. Diabet Med 14: 527-531

5. Selby PL, Jude EB, Burgess J et al. (1998) Bone turnover markers in acute Charcot neuroarthropathy. Diabetologia 41 [Suppl 1]: A275

6. Armstrong DG, Tood WF, Lavery LA, Harkless LB, Bushman TR (1997) The natural history of acute Charcot's arthropathy in a diabetic foot speciality clinic. Diabet Med 14: 357-363

7. Jude EB, Boulton AJM (1999) End stage complications of diabetic neuropathy. Diabetes Rev 7: 395-410

8. Selby PL, Young MJ, Adams JE, Boulton AJM (1994) Bisphosphonate: a new treatment for diabetic Charcot neuroarthropathy. Diabet Med 11: 14-20

9. Young MJ, Boulton AJM, Macleod AF, Williams DRR, Sonksen PH (1993) A multicentre study of the prevalence of diabetic peripheral neuropathy in the UK hospital clinic population. Diabetologia 36: 150-154

10. Ford I, Malik RA, Newrick PG, Preston FE, Ward JD (1992) Relationships between haemostatic factors and capillary morphology in human diabetic neuropathy. Thromb Haemost 68: 628-633

11. Sanders LJ, Murray-Leisure K (1998) Infections of the diabetic foot. In: Abramson C, McCarthy C (eds) Infectious 
diseases of the lower extremity, Williams and Wilkins, Baltimore, pp 193-211

12. Shaw JE, Boulton AJM (1995) The Charcot foot. Foot 5: 65-70

13. Devogelaer JP, Dall'Armellina S, Huaux JP, Nagant de Deuxchaisnes C (1988) Dramatic improvement of intractable reflex sympathetic dystrophy syndrome by intravenous infusions of the second generation bisphosphonate APD. J Bone Miner Res 3 [Suppl 1]: S122

14. Rehman MTA, Clayson AD, Marsh D, Adams H, Cantrill J, Anderson DC (1992) Treatment of reflex sympathetic dystrophy with intravenous pamidronate. Bone 13: 116

15. Adami S, Fossaluzza, Gatti D, Fracassi R, Braga V (1997) Bisphosphonate therapy of reflex sympathetic dystrophy syndrome. Ann Rheum Dis 56: 210-204

16. Fitton A, McTavish D (1991) Pamidronate. Drugs 41: 289-318
17. McGill M, Molyneaux L, Bolton T, Ionnou K, Uren R, Yue DK (2000) Response of Charcot's arthropathy to contact casting: assessment by quantitative techniques. Diabetologia 43: 481-484

18. McCrory JL, Morag E, Norkitis AJ et al. (1998) Healing of Charcot fractures: skin temperature and radiographic correlates. Foot 8: 158-165

19. Haworth CD, Selby PL, Webb AK, Mawer EB, Adams JE, Freemont AJ (1998) Severe bone pain after intravenous pamidronate in adult patients with cystic fibrosis. Lancet 352: $1753-1754$

20. Selby PL (1999) Pamidronate in the treatment of Paget's disease. Bone 24 [Suppl 5]: 575-585

21. Nentelenbos JC, van Ginkel FC, Lips P et al. (1991) Effect of a single infusion of aminohydroxyprolidene on calcium and bone metabolism in healthy volunteers monitored during 2 months. J Clin Endocrinol Metab 72: 223-228 